\title{
On the interference color variations in the Hedychridium roseum species-group (Hymenoptera Chrysididae) from Bulgaria
}

\author{
Franco Strumia ${ }^{1} \&$ Toshko Ljubomirov ${ }^{2}$ \\ 1Pisa Universitymail: franco.strumia@unipi.it - ORCID: https://orcid.org/0000-0002-8496-7790 \\ ${ }^{2}$ Institute of Biodiversity and Ecosystem Research - Bulgarian Academy of Sciences \\ Sofiamail: toshkoljubomirov@gmail.com - ORCID: https://orcid.org/0000-0002-3202-3309
}

\begin{abstract}
A number of the Hedychridium roseum species-group individuals (Hymenoptera Chrysididae), captured mainly in Bulgaria hare here studied. We discovered that the presence/absence on metasoma of red interference color is a variable and unstable feature both in the males and the females of this species-group. The body interference colors are directly connected to the cuticle inner structure and thus to body features. As a consequence, the variable metasoma coloration is a valid taxonomic feature ultimately related to the DNA of single individuals. In the case of Hedychridium roseum species-group the taxonomy is complex and often based on subtle features. A review including also the influence of interference colors is discussed and integrated with the most recent taxonomy. In addition, the presence in Bulgaria of the following six species is confirmed: Hedychridium sculpturatum (Abeille de Perrin, 1877); H. scutellare (Tournier, 1878); H. rossicum Gussakovskij, 1948; H. roseum (Rossi, 1790); H. insulare Balthasar, 1953; H. caputaureum G. Trautmann et W. Trautmann, 1919. The lognormal distribution of above species is entirely positive thus statistic inference shows that in Bulgaria all species of Hedychridium roseum species-group have been captured and studied.
\end{abstract}

KEY WORDS Chrysididae; interference colors; Bulgaria; distribution; Corsica; Sardinia; Tuscany; Genetic.

Received 22.06.2021; accepted 03.12.2021; published online 30.12.2021

\section{INTRODUCTION}

The Hedychridium roseum species-group (Linsenmaier 1959a, b) is one of most complex assemblage of sibling species in the family Chrysididae Latreille, 1802. The taxonomy of the group is difficult and the identification of some species is often in conflict by different authors. Traditionally one significant morphological feature of taxonomic value is the metasoma interference coloration, which often can be metallic-red, caused by the positive light interference, or non-metallic (cutaneous-brown caused by the non-positive interference in visible region of human perception).

In Chrysididae the cuticle interference colors are almost always present and used in taxonomy as color patterns for the body and the appendages. The interference colors are produced by presence in cuticle of reflecting layers (Kroiss et al., 2009).
If the distance between reflecting layers is genetically controlled, large color changes could appears due to the chromosome combinations.

In the majority of Chrysididae a variability of interference colors is considered to be uncommon (apart of a few individuals considered as "anomalous"). Humidity status also changes the cuticle thickness producing a general shift toward greenblue color in wet specimens (Strumia \& Dawah, 2019). If conflicting genes are present in a species, the presence of individuals with a different color is possible and expected. Such as a case is presented and discussed below. Evolutionary selection would cancel such variability toward the most efficient solution. This is apparently not our case.

In earlier studies all individuals of the Hedychridium roseum species-group were usually designated as "Hedychridium roseum", a species with a "clear brown non-metallic metasoma in most 
cases". Similar individuals, but with metallic red metasoma were described in few cases as new species, or even included in different speciesgroups (e.g., Hedychridium mediocrate Linsenmaier, 1959). As a consequence, in most collections all old material was simply grouped under the name of "Hedychridium roseum".

Linsenmaier (1959a, b) divided the group on the basis of metallic or not metallic metasoma. He proposed two species-groups: H. roseum (Rossi, 1790) and H. sculpturatum (Abeille de Perrin, 1877). This unique interpretation caused confusion in taxonomy of $H$. roseum species-group. More recently, Arens (2004, 2010a, 2010b, 2011, 2012, 2014) performed an extended revision of the old " $H$. roseum" speciesgroup, well supported by individual's body morphology, color and sculpture. These results and conclusions are based on the study of almost all available material kept in European collections. Arens' conclusion is the presence in Europe of nine species in the H. roseum species-group (Arens, 2010a), mainly based on body shape, metasoma coloration, size and the kind of cuticle sculpture. In addition, a set of subspecies and synonymies were discussed.

Arens' descriptions are sound and detailed, allowing in most cases a correct identification at species level, provided to take into account a possible variable metasoma coloration.

\section{Interference colors}

Interference colors in insect's cuticle follow from two effects: first, by interference from light diffracted from a periodic structure on cuticle surface and, second, by interference from equidistant reflecting layers inside cuticle. In Aculeate Hymenoptera the second effect is more frequent: the interference colors in the insect's cuticle stems from the reflection of light from a series of neighboring interfaces that are separated by distances comparable with a quarter of the light wavelength. As a result of wave superposition from these reflections, some wavelengths are reflected or transmitted in phase and are therefore reinforced, while others are out of phase and reduced in intensity or cancelled out; the net result is that only certain wavelengths are reflected or transmitted and the surface appears colored (M. Ragaei, 2015). Recently Kroiss et al. (2009) studied the cuticle structure of Hedychrum rutilans Dahlbom, 1854 (a Palearctic species) by means of a SEM microscope, showing that cuticle is a multilayer reflector consisting of about six parallel lamellae with a distance of about $h=185 \mathrm{~nm}$ (see Kroiss et al., 2009: 984, fig. 1). For a positive interference in the reflected light the optical phase difference into reflection from the different layers must be an even integer number of half wavelengths (an uneven number cancel the interference). Thus the following equation must be satisfied: $m \lambda$ $=2 n h \cos (\theta)$, where $m$ is and integer number, $n$ the medium refraction index, $\theta$ the angle with the surface normal, and $h$ the distance between the reflecting layers. By increasing the angle $\theta$ the color of reflected light shifts towards blue. Assuming $h=200 \mathrm{~nm}, m=1, n=1.4$ (about the water refraction index), we obtain $\lambda=560 \mathrm{~nm}$, a bright green reflected interference color. If $h$ increases to $300 \mathrm{~nm}$ the maximum of reflected wavelength move to near infrared $(840 \mathrm{~nm})$ thus producing the non-metallic brown color of cuticle material. The second order interference is in the blue-violet and ultraviolet and can be observed by human eye only as a weak violet shining. When $h$ is too short the interference color is not possible into visible range and the cuticle lose its color showing only those of the cuticle material (color, usually light brown).

The above interpretation is also supported by the observed shift toward green-blue color in the humid individuals, in contrast with dry specimens (Strumia \& Dawah, 2019). It is worth to note that by performing quantitative spectra of reflected light it should be possible to measure the maximum wavelength of first and second order interference and to calculate the structure of reflecting layers on the basis of interference theory.

\section{MATERIAL AND METHODS}

The material from Bulgaria was captured mainly on central Bulgaria hills and it is keep in the Institute of Biodiversity and Ecosystem Research - Bulgarian Academy of Sciences, Sofia (some voucher also into F. Strumia collection). The material from Italy (Tuscany, Piedmont, Sardinia and Corsica) was mainly captured by Malaise Traps or by water plates and is now kept in F. Strumia collection and in the Natural History Museum of Pisa University. The western material was assembled in the frame of the "INTERREG" European research projects. 


\begin{tabular}{|c|c|c|c|c|c|c|}
\hline Sex & Locality & Latitude & Longitude & Date & Collector & $\begin{array}{l}\text { Metasoma } \\
\text { color }\end{array}$ \\
\hline$\hat{\sigma}$ & Bulgaria, south of Petrich & $41^{\circ} 22^{\prime} 05^{\prime \prime} \mathrm{N}$ & $23^{\circ} 11^{\prime} 14^{\prime \prime} \mathrm{E}$ & 30.07 .2002 & O. Todorov & Metallic red \\
\hline 우 & Bulgaria, south of Petrich & $41^{\circ} 22^{\prime} 05^{\prime \prime} \mathrm{N}$ & $23^{\circ} 11^{\prime} 14^{\prime \prime} \mathrm{E}$ & $5-10.08 .2002$ & O. Todorov & Non-metallic \\
\hline 우 & Bulgaria, Plovdiv & $42^{\circ} 08^{\prime} 06^{\prime \prime} \mathrm{N}$ & $24^{\circ} 45^{\prime} 58^{\prime \prime} \mathrm{E}$ & $8-22.08 .2002$ & S. Petrov & Non-metallic \\
\hline 우 & Bulgaria, Plovdiv & $42^{\circ} 08^{\prime} 06^{\prime \prime} \mathrm{N}$ & $24^{\circ} 45^{\prime} 58^{\prime \prime} \mathrm{E}$ & $8-22.08 .2002$ & S. Petrov & Non-metallic \\
\hline 우 & Bulgaria, Plovdiv & $42^{\circ} 08^{\prime} 06^{\prime \prime} \mathrm{N}$ & $24^{\circ} 45^{\prime} 58^{\prime \prime} \mathrm{E}$ & $17-25.08 .1999$ & S. Petrov & Non-metallic \\
\hline 우 & Bulgaria, south-west of Borika & $42^{\circ} 28^{\prime} 55^{\prime \prime} \mathrm{N}$ & $23^{\circ} 36^{\prime} 40^{\prime \prime} \mathrm{E}$ & 6.08 .2010 & T. Ljubomirov & Non-metallic \\
\hline 우 & Bulgaria, sout & $42^{\circ} 28^{\prime} 55^{\prime \prime} \mathrm{N}$ & $23^{\circ} 36^{\prime} 40^{\prime \prime} \mathrm{E}$ & 6.08 .2010 & T. Ljubomirov & Non-metallic \\
\hline 우 & Bulgaria, $\mathrm{r}$ & $42^{\circ} 40^{\prime} 50^{\prime \prime} \mathrm{N}$ & $25^{\circ} 24^{\prime} 57^{\prime \prime} \mathrm{E}$ & 23-29.07.2000 & M. Langourov & Non-n \\
\hline 우 & st of Zemen & $42^{\circ} 28^{\prime} 19^{\prime \prime} \mathrm{N}$ & $22^{\circ} 44^{\prime} 06^{\prime \prime} \mathrm{E}$ & 13.07 .2006 & mirov & Non-1 \\
\hline 우 & Italy, San Pietro Belvedere, Pisa & $43^{\circ} 34^{\prime} 19^{\prime \prime} \mathrm{N}$ & $10^{\circ} 39^{\prime} 56^{\prime \prime} \mathrm{E}$ & $19-25.08 .1998$ & F. Strumia & Non-metallic \\
\hline 우 & Italy, San Pietro Belvedere, Pisa & $43^{\circ} 34^{\prime} 19^{\prime \prime} \mathrm{N}$ & $10^{\circ} 39^{\prime} 56^{\prime \prime} \mathrm{E}$ & 27-30.07.1998 & F. Strumia & Non-metallic \\
\hline 우 & Italy, $\mathrm{n}$ & $43^{\circ} 40^{\prime} 31^{\prime \prime} \mathrm{N}$ & $10^{\circ} 19^{\prime} 36^{\prime \prime} \mathrm{E}$ & $26.07-05.08 .1998$ & F. Strumia & Non-1 \\
\hline 우 & Italy, Chianni, Pisa & $43^{\circ} 27^{\prime} 50^{\prime \prime} \mathrm{N}$ & $10^{\circ} 40^{\prime} 44^{\prime \prime} \mathrm{E}$ & $16-22.08 .2000$ & F. Strumia & Non-metallic \\
\hline 우 & Italy, Chianni, Pisa & $43^{\circ} 27^{\prime} 50^{\prime \prime} \mathrm{N}$ & $10^{\circ} 40^{\prime} 44^{\prime \prime} \mathrm{E}$ & 3-11.08.1998 & F. Strumia & Non-metallic \\
\hline 우 & Italy, San Pietro Belvedere, Pisa & $43^{\circ} 34^{\prime} 19^{\prime \prime} \mathrm{N}$ & $10^{\circ} 39^{\prime} 56^{\prime \prime} \mathrm{E}$ & $19-25.08 .1998$ & F. Strumia & Non-metallic \\
\hline 우 & Italy, near Pisa & $43^{\circ} 40^{\prime} 30^{\prime \prime} \mathrm{N}$ & $10^{\circ} 19^{\prime} 36^{\prime \prime} \mathrm{E}$ & $9-19.09 .1998$ & F. Strumia & Non-metallic \\
\hline 우 & Italy, Chi & $3^{\circ} 27^{\prime} 50^{\prime \prime} \mathrm{N}$ & $10^{\circ} 40^{\prime} 44^{\prime \prime} \mathrm{E}$ & .2000 & F. Strumia & Non- \\
\hline 우 & Italy, Piombino & $42^{\circ} 56^{\prime} 44^{\prime \prime} \mathrm{N}$ & $10^{\circ} 29^{\prime} 56^{\prime \prime} \mathrm{E}$ & $21.08-01.092000$ & F. Strumia & Non-metallic \\
\hline 우 & Italy, Piombino & $42^{\circ} 56^{\prime} 44^{\prime \prime} \mathrm{N}$ & $10^{\circ} 29^{\prime} 56^{\prime \prime} \mathrm{E}$ & $11-21.082000$ & F. Strumia & Non-metallic \\
\hline 우 & Italy, San Pietro Belvedere, Pisa & $43^{\circ} 34^{\prime} 19^{\prime \prime} \mathrm{N}$ & $10^{\circ} 39^{\prime} 56^{\prime \prime} \mathrm{E}$ & 26.07-05.08. 1998 & F. Strumia & Non-metallic \\
\hline 우 & Italy, San Pietro Belv & $43^{\circ} 34^{\prime} 19^{\prime \prime} \mathrm{N}$ & $10^{\circ} 39^{\prime} 56^{\prime \prime} \mathrm{E}$ & 15.08 .1998 & F. Stru & Metallic red \\
\hline 우 & Italy, San Pietro Belvedere, Pisa & $43^{\circ} 34^{\prime} 19^{\prime \prime} \mathrm{N}$ & $10^{\circ} 39^{\prime} 56^{\prime \prime} \mathrm{E}$ & $25-30.07 .1998$ & F. Strumia & Non-metallic \\
\hline 우 & Italy, San Pietro Belvedere, Pisa & $43^{\circ} 34^{\prime} 19^{\prime \prime} \mathrm{N}$ & $10^{\circ} 39^{\prime} 56^{\prime \prime} \mathrm{E}$ & $19-25.08 .1998$ & F. Strumia & Non-metallic \\
\hline 우 & Italy, San Pietro Belvedere, Pisa & $43^{\circ} 34^{\prime} 19^{\prime \prime} \mathrm{N}$ & $10^{\circ} 39^{\prime} 56^{\prime \prime} \mathrm{E}$ & 19-25.08. 1998 & F. Strumia & Non-metallic \\
\hline 우 & Italy, Piombino, Livorno & $42^{\circ} 56^{\prime} 44^{\prime \prime} \mathrm{N}$ & $0^{\circ} 29^{\prime} 56^{\prime \prime} \mathrm{E}$ & $21.08-11.09 .2000$ & F. Strumia & Non-metallic \\
\hline 우 & Italy, Piombino, Livorno & $42^{\circ} 56^{\prime} 44^{\prime \prime} \mathrm{N}$ & $10^{\circ} 29^{\prime} 56^{\prime \prime} \mathrm{E}$ & $11-21.08 .2000$ & F. Strumia & Non-metallic \\
\hline 우 & Italy, Pisa & $43^{\circ} 40^{\prime} 30^{\prime \prime} \mathrm{N}$ & $10^{\circ} 19^{\prime} 36^{\prime \prime} \mathrm{E}$ & 26.07-05.08. 1998 & F. Strumia & Non-metallic \\
\hline 우 & Italy, Pisa & $43^{\circ} 40^{\prime} 30^{\prime \prime} \mathrm{N}$ & $10^{\circ} 19^{\prime} 36^{\prime \prime} \mathrm{E}$ & 26.07-05.08. 1998 & F. Strumia & Non-metallic \\
\hline 우 & Italy, Pisa & $43^{\circ} 40^{\prime} 30^{\prime \prime} \mathrm{N}$ & $10^{\circ} 19^{\prime} 36^{\prime \prime} \mathrm{E}$ & $9-19.09 .1998$ & F. Strumia & Non-metallic \\
\hline 우 & Italy, Chianni, Pisa & $43^{\circ} 27^{\prime} 50^{\prime \prime} \mathrm{N}$ & $10^{\circ} 40^{\prime} 44^{\prime \prime} \mathrm{E}$ & $3-11.08 .2000$ & F. Strumia & Non-metallic \\
\hline 우 & Italy, Chianni, Pisa & $43^{\circ} 27^{\prime} 50^{\prime \prime} \mathrm{N}$ & $10^{\circ} 40^{\prime} 44^{\prime \prime} \mathrm{E}$ & $11-16.08 .2000$ & F. Strumia & Non-metallic \\
\hline 우 & Italy, Chianni, Pisa & $43^{\circ} 27^{\prime} 50^{\prime \prime} \mathrm{N}$ & $10^{\circ} 40^{\prime} 44^{\prime \prime} \mathrm{E}$ & $16-22.08 .2000$ & F. Strumia & Non-metallic \\
\hline$\hat{\sigma}$ & Italy, Piom & $42^{\circ} 56^{\prime} 44^{\prime \prime} \mathrm{N}$ & $10^{\circ} 29^{\prime} 56^{\prime \prime} \mathrm{E}$ & $4-13.07 .2$ & F. Strumia & Non- \\
\hline$\hat{\sigma}$ & Italy, Montefalcone Nat. Res. Pisa & $43^{\circ} 44^{\prime} 40^{\prime \prime} \mathrm{N}$ & $10^{\circ} 43^{\prime} 10^{\prime \prime} \mathrm{E}$ & 20-30.07.2000 & F. Strumia & Metallic red \\
\hline$\hat{0}$ & Italy, Piombino & $42^{\circ} 56^{\prime} 44^{\prime \prime} \mathrm{N}$ & $10^{\circ} 29^{\prime} 56^{\prime \prime} \mathrm{E}$ & 20.05.-03.06.2000 & F. Strumia & Metallic red \\
\hline$\hat{\sigma}$ & Italy, Piombino & $42^{\circ} 56^{\prime} 44^{\prime \prime} \mathrm{N}$ & $10^{\circ} 29^{\prime} 56^{\prime \prime} \mathrm{E}$ & $4.07-13.07 .2000$ & F. Strumia & Metallic red \\
\hline$\hat{\sigma}$ & Italy, San Pietro Belvedere & $43^{\circ} 34^{\prime} 19^{\prime \prime} \mathrm{N}$ & $10^{\circ} 39^{\prime} 56^{\prime \prime} \mathrm{E}$ & 9-15.08.1998 & F. Strumia & Non-metallic \\
\hline$\hat{\sigma}$ & Italy, Piombino, Livorno & $42^{\circ} 56^{\prime} 44^{\prime \prime} \mathrm{N}$ & $10^{\circ} 29^{\prime} 56^{\prime \prime} \mathrm{E}$ & $4.07-13.08 .2000$ & F. Strumia & Metallic red \\
\hline$\hat{\sigma}$ & Italy, Piombino, Livorno & $42^{\circ} 56^{\prime} 44^{\prime \prime} \mathrm{N}$ & $10^{\circ} 29^{\prime} 56^{\prime \prime} \mathrm{E}$ & $4.07-13.08 .2000$ & F. Strumia & Metallic red \\
\hline$\widehat{0}$ & Italy, Montefalcone Nat. Res. Pisa & $42^{\circ} 44^{\prime} 40^{\prime \prime} \mathrm{N}$ & $10^{\circ} 43^{\prime} 10^{\prime \prime} \mathrm{E}$ & 20.07-30.07.2000 & F. Strumia & Metallic red \\
\hline 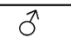 & Italy, Piombino & $42^{\circ} 56^{\prime} 44^{\prime \prime} \mathrm{N}$ & $10^{\circ} 29^{\prime} 56^{\prime \prime} \mathrm{E}$ & 20.05-03.06.2000 & F. Strumia & Metallic red \\
\hline
\end{tabular}

Table 1. Data on the studied individuals of Hedychridium sculpturatum. 


\section{RESULTS}

Recently it was possible to capture and study a large number of $H$. roseum species-group individuals in Bulgaria (by T. Ljubomirov), in Italy, Sardinia and Corsica (by F. Strumia). In addition, few more specimens from Greece, Turkey and Iran were available. It is thus possible to study the distribution of metasoma interference color, at least in the most frequently met species. Details on the origin of the material and the distribution of interference color on metasoma are presented in the following Tables.

In Bulgaria, over 120 individuals of H. roseum species-group were captured and identified on the basis of Arens' descriptions (Arens, 2012). Below, we confirm the presence of six species of this group.

\section{Data on studied species}

Hedychridium sculpturatum (Abeille de Perrin, 1877)

= Hedychridium sculpturatum pseudoroseum Linsenmaier, 1959

= Hedychridium placare Linsenmaier, 1968

= Hedychridium lampadum austeritatum Linsenmaier, 1997

Distribution. South Europe, Cyprus, Ukraine, Turkey, Russia, North Caucasus, Near East, Kirghizstan, Mongolia, Western Siberia (Arens, 2010a, 2012).

REMARKS ON COLORATION. In Bulgaria we captured 8 females with non-metallic metasoma and only one male (identified by the studied genital apparatus) with metallic red metasoma. In Italy, in addition, we studied 32 individuals of $H$. sculpturatum. The metasoma color distribution results in $31 \mathrm{fe}-$ males and 2 males having non-metallic metasoma and 1 female and 7 males having metallic red metasoma. In Italy only one female has the metallic red metasoma and only two males have non-metallic metasoma.

We can suppose that the majority of $H$. sculpturatum is sexually dimorphic (as noted by Arens): females mostly with non-metallic red metasoma and males mostly with metallic red metasoma. It is worth mentioning that the single female with red metasoma had been captured in Tuscany.

In Table 1 are shown the data of individuals of H. sculpturatum studied by us.
Hedychridium scutellare (Tournier, 1878)

= Hedychridium scutellare palestinense Balthasar, 1953

= Hedychridium mediocre Linsenmaier, 1959

= Hedychridium mediocre sardiniense Linsenmaier, 1959

= Hedychridium scutellare maculiventre Linsenmaier, 1959

= Hedychridium scutellare sculpturatissimum Linsenmaier, 1959

= Hedychridium mediocre corsuense Perraudin, 1978

= Hedychridium tunesiense Linsenmaier, 1959

= Hedychridium maculiventre raucum Linsenmaier, 1997

= Hedychridium sardinum Linsenmaier, 1997

Distribution. South Europe from Portugal to Croatia, Greece, Russia, north Caucasus (Tyrner, 1991; Rosa et al., 2019).

Remarks on coloration. Four females from Bulgaria have non-metallic metasoma and only one female from Greece show a metallic red metasoma. No male individuals of $H$. scutellare from Bulgaria could be studied. More individuals are needed to prove the sexual dimorphism in the metasoma interference color. From Italy we could study only 3 females and 1 male, all individuals have metallic red metasoma. Two males and $1 \mathrm{fe}-$ male from Iran (in F. Strumia collection) have metallic red metasoma. Apparently, in H. scutellare both females and males have metallic red metasoma. The studied material is listed in Table 2.

Hedychridium scutellare is a new record for Bulgaria.

Hedychridium rossicum Gussakovskij, 1948

$=$ Hedychridium valesiense Linsenmaier, 1959

= Hedychridium valesiense turcicum Arens, 2010

Distribution. South and central Europe, Ukraine, Near East, Moldova, Russia (Orenburg Province, Sverdlovsk Province), Crimea (Arens, 2010a; Vinokurov, 2013; Martynova, 2015). H. rossicum is the most frequent species in Bulgaria, Italy and, perhaps, in south Europe as a whole.

REMARKS ON COLORATION. Arens (2010a) studied over 120 males of $H$. rossicum, including 


\begin{tabular}{|l|l|l|l|l|l|l|}
\hline Sex & Locality & Latitude & Longitude & Date & Collector & $\begin{array}{l}\text { Metasoma } \\
\text { color }\end{array}$ \\
\hline$q$ & Iran, Bam & $29^{\circ} 06^{\prime} 02^{\prime \prime} \mathrm{N}$ & $58^{\circ} 19^{\prime} 44^{\prime \prime} \mathrm{E}$ & $22.05-4.07 .2017$ & M. Fallahzadeh & Metallic red \\
\hline$q$ & Iran, Bam & $29^{\circ} 06^{\prime} 02^{\prime \prime} \mathrm{N}$ & $58^{\circ} 19^{\prime} 44^{\prime \prime} \mathrm{E}$ & $22.05-4.07 .2017$ & M. Fallahzadeh & Metallic red \\
\hline$\sigma^{\lambda}$ & Italy, Barbaresco (Cuneo) & $44^{\circ} 43^{\prime} 26^{\prime \prime} \mathrm{N}$ & $8^{\circ} 04^{\prime} 26^{\prime \prime} \mathrm{E}$ & 26.07 .2017 & F. Strumia & Metallic red \\
\hline$q$ & Bulgaria, north of Enina & $42^{\circ} 40^{\prime} 50^{\prime \prime} \mathrm{N}$ & $25^{\circ} 24^{\prime} 57^{\prime \prime} \mathrm{E}$ & $23-29.07 .2000$ & M. Langourov & Non-metallic \\
\hline$q$ & Bulgaria, south of Vitanovo & $42^{\circ} 03^{\prime} 34^{\prime \prime} \mathrm{N}$ & $27^{\circ} 25^{\prime} 14^{\prime \prime} \mathrm{E}$ & $24.07-24.08 .1999$ & S. Petrov & Metallic red \\
\hline$q$ & Bulgaria, south of Brushlyan & $42^{\circ} 03^{\prime} 34^{\prime \prime} \mathrm{N}$ & $27^{\circ} 25^{\prime} 14^{\prime \prime} \mathrm{E}$ & $8.06-24.07 .1999$ & S. Petrov & Metallic red \\
\hline$q$ & Greece, north-east of Liri & $39^{\circ} 12^{\prime} 06^{\prime \prime} \mathrm{N}$ & $23^{\circ} 20^{\prime} 15^{\prime \prime} \mathrm{E}$ & 9.09 .2014 & T. Ljubomirov & Metallic red \\
\hline$q$ & Bulgaria, west of Vulkovo & $41^{\circ} 34^{\prime} 39^{\prime \prime} \mathrm{N}$ & $23^{\circ} 12^{\prime} 51^{\prime \prime} \mathrm{E}$ & 4.10 .2003 & T. Ljubomirov & Non-metallic \\
\hline+ & Iran, south of Jiroft & $28^{\circ} 41^{\prime} 07^{\prime \prime} \mathrm{N}$ & $57^{\circ} 55^{\prime} 17^{\prime \prime} \mathrm{E}$ & $20.04-5.05 .2017$ & M. Fallahzadeh & Metallic red \\
\hline$q$ & Italy, north-west of Piombino & $42^{\circ} 56^{\prime} 02^{\prime \prime} \mathrm{N}$ & $10^{\circ} 29^{\prime} 44^{\prime \prime} \mathrm{E}$ & $11-21.08 .2000$ & F. Strumia & Metallic red \\
\hline$q$ & Italy, north-west of Piombino & $42^{\circ} 56^{\prime} 02^{\prime \prime} \mathrm{N}$ & $10^{\circ} 29^{\prime} 44^{\prime \prime} \mathrm{E}$ & $11-21.08 .2000$ & F. Strumia & Metallic red \\
\hline$q$ & Italy, north-west of Piombino & $42^{\circ} 56^{\prime} 02^{\prime \prime} \mathrm{N}$ & $10^{\circ} 29^{\prime} 44^{\prime \prime} \mathrm{E}$ & $11-21.08 .2000$ & F. Strumia & Metallic red \\
\hline
\end{tabular}

Table 2. Data on the studied individuals of Hedychridium scutellare.

the holotype and in addition,reported two males from Italy (Aosta-Valley, Ozein 1000m, 05.08.1991, 2.7.1994, leg. P. Rosa) with metasoma partlially metallic green (Figs. 1-4). From Bulgaria we captured 75 individuals, 43 females and 28 males of $H$. rossicum, mainly on the mountains of central Bulgaria. The sexual dimorphism in this species is minimal and males and females are easily identified according the descriptions of Arens (2010a, 2010b). In addition, the genitalia of all males were studied to confirm the identifications. In all previous investigations it was stated that males have the metasoma dorsum metallic red by interference and females the metasoma non-metallic brown (Figs. 1-4).

We discover that this statement is variable since we found 5 males with non-metallic brown metasoma (about $20 \%$ of studied males) as in females - see Fig. 2. We also found two males (about $5 \%$ ) with bicolor metasoma (one from Bulgaria, one from Italy) (Fig. 4). From Italy (Tuscany) we could study 18 additional individuals of $H$. rossicum with the following result: 13 females with non-metallic metasoma, 2 males with metallic red metasoma and 3 males with non-metallic metasoma. Similar result is obtained from the study of additional 26 individuals captured in Corsica Island: 17 females having non-metallic metasoma against 2 males having metallic red metasoma and 7 males having non-metallic metasoma. It follows that almost all studied females of $H$. rossicum have a non-metallic metasoma. On the contrary the males have either a red metallic metasoma or a non-metallic metasoma in a variable ratio, contrary to older studies (in addition 2 males with a mixed bicolor red and green metasoma, as shown in Figs. 1-4).

Hedychridium caputaureum G. Trautmann et W. Trautmann, 1919

= Hedychridium chloropygum densum Linsenmaier, 1959

= Hedychridium chloropygum spatium Linsenmaier, 1959

= Hedychridium chloropygum ottomanum Arens, 2010

Distribution. North and central Europe, Ukraine, Asia Minor, Russia, north Caucasus (Arens, 2010a; Table 4 Data of the studied individuals of $H$. capuraureum.

REMARKS ON COLORATION. In general, 11 females and one male of $H$. caputaureum were captured. They come from Bulgaria and Turkey. All females show non-metallic metasoma, while the single male has a red metallic metasoma. Study of more males is needed to establish the fraction of dimorphic metasoma color. 


\begin{tabular}{|c|c|c|c|c|c|c|}
\hline Sex & Locality & Latitude & Longitude & Date & Collector 11 & $\begin{array}{l}\text { Metasoma } \\
\text { color }\end{array}$ \\
\hline$\hat{0}^{\lambda}$ & Bulgaria, north-west of Passarel & $42^{\circ} 33^{\prime} 05^{\prime \prime} \mathrm{N}$ & $23^{\circ} 29^{\prime} 30^{\prime \prime} \mathrm{E}$ & 9.08 .1995 & T. Ljubomirov & Metallic red \\
\hline q & Bulgaria, north-west of Passarel & $42^{\circ} 33^{\prime} 05^{\prime \prime} \mathrm{N}$ & $23^{\circ} 29^{\prime} 30^{\prime \prime} \mathrm{E}$ & 4.07 .2013 & T. Ljubomirov & Non-metallic \\
\hline$\hat{\sigma}$ & Bulgaria, north-west of Passarel & $42^{\circ} 33^{\prime} 05^{\prime \prime} \mathrm{N}$ & $23^{\circ} 29^{\prime} 30^{\prime \prime} \mathrm{E}$ & 29.06 .2006 & T. Ljubomirov & Metallic red \\
\hline q & Bulgaria, north-west of Passarel & $42^{\circ} 33^{\prime} 05^{\prime \prime} \mathrm{N}$ & $23^{\circ} 29^{\prime} 30^{\prime \prime} \mathrm{E}$ & 24.07 .2002 & T. Ljubomirov & Non-metallic \\
\hline$\hat{0}$ & Bulgaria, south-west of Barutin & $41^{\circ} 34^{\prime} 08^{\prime \prime} \mathrm{N}$ & $24^{\circ} 10^{\prime} 08^{\prime \prime} \mathrm{E}$ & 7.08 .2018 & T. Ljubomirov & Metallic red \\
\hline$\hat{0}$ & Bulgaria, south-west of Barutin & $41^{\circ} 34^{\prime} 08^{\prime \prime} \mathrm{N}$ & $24^{\circ} 10^{\prime} 08^{\prime \prime} \mathrm{E}$ & 7.08 .2018 & T. Ljubomirov & Non-metallic \\
\hline$\hat{0}$ & Bulgaria, east of Kladnitsa & $42^{\circ} 33^{\prime} 13^{\prime \prime} \mathrm{N}$ & $23^{\circ} 11^{\prime} 39^{\prime \prime} \mathrm{E}$ & 4.07 .1998 & T. Ljubomirov & Metallic red \\
\hline$\hat{0}$ & Bulgaria, west of Zheleznitsa & $42^{\circ} 32^{\prime} 06^{\prime \prime} \mathrm{N}$ & $23^{\circ} 21^{\prime} 08^{\prime \prime} \mathrm{E}$ & 14.07.1999 & T. Ljubomirov & $\begin{array}{l}\text { Metallic red- } \\
\text { green }\end{array}$ \\
\hline q & Bulgaria, west of Etropole & $42^{\circ} 50^{\prime} 29^{\prime \prime} \mathrm{N}$ & $23^{\circ} 58^{\prime} 20^{\prime \prime} \mathrm{E}$ & 17.09 .2002 & T. Ljubomirov & Non-metallic \\
\hline$\hat{0}$ & Bulgaria, north of Bisstritsa & $42^{\circ} 35^{\prime} 41^{\prime \prime} \mathrm{N}$ & $23^{\circ} 20^{\prime} 30^{\prime \prime} \mathrm{E}$ & 5.08 .1998 & T. Ljubomirov & Metallic red \\
\hline$\hat{\sigma}$ & Bulgaria, north of Bisstritsa & $42^{\circ} 36^{\prime} 03^{\prime \prime} \mathrm{N}$ & $23^{\circ} 20^{\prime} 13^{\prime \prime} \mathrm{E}$ & 5.08 .1998 & T. Ljubomirov & Metallic red \\
\hline$\hat{0}$ & Bulgaria, north of Bisstritsa & $42^{\circ} 36^{\prime} 03^{\prime \prime} \mathrm{N}$ & $23^{\circ} 20^{\prime} 13^{\prime \prime} \mathrm{E}$ & 5.08 .1998 & T. Ljubomirov & Metallic red \\
\hline$\hat{\sigma}$ & Bulgaria, north of Tchuypetlovo & $42^{\circ} 31^{\prime} 43^{\prime \prime} \mathrm{N}$ & $23^{\circ} 14^{\prime} 08^{\prime \prime} \mathrm{E}$ & 22.07 .1998 & T. Ljubomirov & $\begin{array}{l}\text { Mixe metallic } \\
\text { red-green }\end{array}$ \\
\hline$\hat{0}$ & Bulgaria, north of Varna & $43^{\circ} 14^{\prime} 05^{\prime \prime} \mathrm{N}$ & $28^{\circ} 01^{\prime} 14^{\prime \prime} \mathrm{E}$ & 22.07 .2001 & I. Todorov & Metallic red \\
\hline$\hat{0}$ & Bulgaria, west of Gorna Breznitsa & $41^{\circ} 44^{\prime} 24^{\prime \prime} \mathrm{N}$ & $23^{\circ} 06^{\prime} 01^{\prime \prime} \mathrm{E}$ & 10.07 .2013 & T. Ljubomirov & Metallic red \\
\hline$\hat{0}$ & Bulgaria, south-east of Ardino & $41^{\circ} 34^{\prime} 38^{\prime \prime} \mathrm{N}$ & $25^{\circ} 08^{\prime} 42^{\prime \prime} \mathrm{E}$ & 15.08 .2016 & T. Ljubomirov & Metallic red \\
\hline q & Bulgaria, west of Milanovo & $43^{\circ} 05^{\prime} 40^{\prime \prime} \mathrm{N}$ & $23^{\circ} 23^{\prime} 23^{\prime \prime} \mathrm{E}$ & 7.07 .2015 & T. Ljubomirov & Non-metallic \\
\hline$\hat{\sigma}$ & Bulgaria, Muglizh & $42^{\circ} 36^{\prime} 14^{\prime \prime} \mathrm{N}$ & $25^{\circ} 32^{\prime} 40^{\prime \prime} \mathrm{E}$ & $17-24.06 .1995$ & I. Stoyanov & Metallic red \\
\hline$\hat{0}$ & Bulgaria, Muglizh & $42^{\circ} 36^{\prime} 14^{\prime \prime} \mathrm{N}$ & $25^{\circ} 32^{\prime} 40^{\prime \prime} \mathrm{E}$ & $17-24.06 .1995$ & I. Stoyanov & Metallic red \\
\hline$\hat{0}$ & Bulgaria, south of Petrich & $41^{\circ} 22^{\prime} 05^{\prime \prime} \mathrm{N}$ & $23^{\circ} 11^{\prime} 14^{\prime \prime} \mathrm{E}$ & $10-20.07 .2002$ & O. Todorov & Metallic red \\
\hline$q$ & Bulgaria, west of Etropole & $42^{\circ} 49^{\prime} 55^{\prime \prime} \mathrm{N}$ & $23^{\circ} 57^{\prime} 44^{\prime \prime} \mathrm{E}$ & 1.07 .2010 & T. Ljubomirov & Non-metallic \\
\hline$\hat{\sigma}$ & Bulgaria, west of Etropole & $42^{\circ} 49^{\prime} 55^{\prime \prime} \mathrm{N}$ & $23^{\circ} 57^{\prime} 44^{\prime \prime} \mathrm{E}$ & 1.07 .2010 & T. Ljubomirov & Metallic red \\
\hline$\hat{\sigma}$ & Bulgaria, west of Etropole & $42^{\circ} 49^{\prime} 55^{\prime \prime} \mathrm{N}$ & $23^{\circ} 57^{\prime} 44^{\prime \prime} \mathrm{E}$ & 1.07 .2010 & T. Ljubomirov & Metallic red \\
\hline$\hat{\sigma}$ & Bulgaria, west of Etropole & $42^{\circ} 49^{\prime} 55^{\prime \prime} \mathrm{N}$ & $23^{\circ} 57^{\prime} 44^{\prime \prime} \mathrm{E}$ & 1.07 .2010 & T. Ljubomirov & Metallic red \\
\hline$q$ & Bulgaria, north-west of Passarel & $42^{\circ} 33^{\prime} 05^{\prime \prime} \mathrm{N}$ & $23^{\circ} 29^{\prime} 30^{\prime \prime} \mathrm{E}$ & 21.06 .1997 & T. Ljubomirov & Non-metallic \\
\hline$q$ & Bulgaria, north-west of Passarel & $42^{\circ} 33^{\prime} 05^{\prime \prime} \mathrm{N}$ & $23^{\circ} 29^{\prime} 30^{\prime \prime} \mathrm{E}$ & 21.06 .1997 & T. Ljubomirov & Non-metallic \\
\hline q & Bulgaria, north-west of Passarel & $42^{\circ} 33^{\prime} 05^{\prime \prime} \mathrm{N}$ & $23^{\circ} 29^{\prime} 30^{\prime \prime} \mathrm{E}$ & 21.06 .1997 & T. Ljubomirov & Non-metallic \\
\hline
\end{tabular}




\begin{tabular}{|c|c|c|c|c|c|c|}
\hline 우 & Bulgaria, north-west of Passarel & $42^{\circ} 33^{\prime} 05^{\prime \prime} \mathrm{N}$ & $23^{\circ} 29^{\prime} 30^{\prime \prime} \mathrm{E}$ & 4.07 .2013 & T. Ljubomirov & Non-metallic \\
\hline 우 & Bulgaria, north of Enina & $42^{\circ} 40^{\prime} 50^{\prime \prime} \mathrm{N}$ & $25^{\circ} 24^{\prime} 57^{\prime \prime} \mathrm{E}$ & $23-29.07 .2000$ & M. Langourov & Non-metallic \\
\hline 우 & Bulgaria, east of Kladnitsa & $42^{\circ} 33^{\prime} 29^{\prime \prime} \mathrm{N}$ & $23^{\circ} 12^{\prime} 12^{\prime \prime} \mathrm{E}$ & 25.08 .2000 & T. Ljubomirov & Non-metallic \\
\hline 우 & Bulgaria, east of Kladnitsa & $42^{\circ} 33^{\prime} 29^{\prime \prime} \mathrm{N}$ & $23^{\circ} 12^{\prime} 12^{\prime \prime} \mathrm{E}$ & 25.08 .2000 & T. Ljubomirov & Non-metallic \\
\hline 우 & Bulgaria, east of Kladnitsa & $42^{\circ} 33^{\prime} 29^{\prime \prime} \mathrm{N}$ & $23^{\circ} 12^{\prime} 12^{\prime \prime} \mathrm{E}$ & 25.08 .2000 & T. Ljubomirov & Non-metallic \\
\hline 우 & Bulgaria, east of Kladnitsa & $42^{\circ} 33^{\prime} 29^{\prime \prime} \mathrm{N}$ & $23^{\circ} 12^{\prime} 12^{\prime \prime} \mathrm{E}$ & 25.08 .2000 & T. Ljubomirov & Non-metallic \\
\hline 우 & Bulgaria, east of Kladnitsa & $42^{\circ} 33^{\prime} 13^{\prime \prime} \mathrm{N}$ & $23^{\circ} 11^{\prime} 39^{\prime \prime} \mathrm{E}$ & 4.09 .1998 & T. Ljubomirov & Non-metallic \\
\hline 우 & Bulgaria, east of Kladnitsa & $42^{\circ} 33^{\prime} 14^{\prime \prime} \mathrm{N}$ & $23^{\circ} 11^{\prime} 24^{\prime \prime} \mathrm{E}$ & 22.07.1998 & T. Ljubomirov & Non-metallic \\
\hline 우 & Bulgaria, west of Zheleznitsa & $42^{\circ} 32^{\prime} 06^{\prime \prime} \mathrm{N}$ & $23^{\circ} 21^{\prime} 08^{\prime \prime} \mathrm{E}$ & 12.09 .2000 & T. Ljubomirov & Non-metallic \\
\hline 우 & Bulgaria, west of Zheleznitsa & $42^{\circ} 32^{\prime} 07^{\prime \prime} \mathrm{N}$ & $23^{\circ} 20^{\prime} 48^{\prime \prime} \mathrm{E}$ & 12.09 .2000 & T. Ljubomirov & Non-metallic \\
\hline 우 & Bulgaria, west of Zheleznitsa & $42^{\circ} 32^{\prime} 06^{\prime \prime} \mathrm{N}$ & $23^{\circ} 21^{\prime} 04^{\prime \prime} \mathrm{E}$ & 24.08 .1998 & T. Ljubomirov & Non-metallic \\
\hline$\hat{\sigma}$ & Bulgaria, west of Milanovo & $43^{\circ} 05^{\prime} 40^{\prime \prime} \mathrm{N}$ & $23^{\circ} 23^{\prime} 23^{\prime \prime} \mathrm{E}$ & 7.07.2015 & T. Ljubomirov & Metallic red \\
\hline 우 & Bulgaria, south of Sofia & $42^{\circ} 38^{\prime} 10^{\prime \prime} \mathrm{N}$ & $23^{\circ} 14^{\prime} 38^{\prime \prime} \mathrm{E}$ & 14.08 .2000 & T. Ljubomirov & Non-metallic \\
\hline 우 & Bulgaria, south of Sofia & $42^{\circ} 38^{\prime} 10^{\prime \prime} \mathrm{N}$ & $23^{\circ} 14^{\prime} 38^{\prime \prime} \mathrm{E}$ & 14.08 .2000 & T. Ljubomirov & Non-metallic \\
\hline 우 & Bulgaria, south of Sofia & $42^{\circ} 38^{\prime} 10^{\prime \prime} \mathrm{N}$ & $23^{\circ} 14^{\prime} 38^{\prime \prime} \mathrm{E}$ & 14.08 .2000 & T. Ljubomirov & Non-metallic \\
\hline 우 & Bulgaria, south of Sofia & $42^{\circ} 38^{\prime} 10^{\prime \prime} \mathrm{N}$ & $23^{\circ} 14^{\prime} 38^{\prime \prime} \mathrm{E}$ & 14.08 .2000 & T. Ljubomirov & Non-metallic \\
\hline q & Bulgaria, south of Sofia & $42^{\circ} 38^{\prime} 10^{\prime \prime} \mathrm{N}$ & $23^{\circ} 14^{\prime} 38^{\prime \prime} \mathrm{E}$ & 14.08 .2000 & T. Ljubomirov & Non-metallic \\
\hline 우 & Bulgaria, south of Kokalyane & $42^{\circ} 34^{\prime} 19^{\prime \prime} \mathrm{N}$ & $23^{\circ} 24^{\prime} 46^{\prime \prime} \mathrm{E}$ & 20.09 .2001 & T. Ljubomirov & Non-metallic \\
\hline 우 & Bulgaria, south of Kokalyane & $42^{\circ} 34^{\prime} 19^{\prime \prime} \mathrm{N}$ & $23^{\circ} 24^{\prime} 46^{\prime \prime} \mathrm{E}$ & 20.09 .2001 & T. Ljubomirov & Non-metallic \\
\hline 우 & Bulgaria, south of Kokalyane & $42^{\circ} 34^{\prime} 19^{\prime \prime} \mathrm{N}$ & $23^{\circ} 24^{\prime} 46^{\prime \prime} \mathrm{E}$ & 20.09 .2001 & T. Ljubomirov & Non-metallic \\
\hline 우 & Bulgaria, Plovdiv & $42^{\circ} 08^{\prime} 06^{\prime \prime} \mathrm{N}$ & $24^{\circ} 45^{\prime} 58^{\prime \prime} \mathrm{E}$ & $17-25.08 .1999$ & S. Petrov & Non-metallic \\
\hline 우 & Bulgaria, north of Tchuypetlovo & $42^{\circ} 31^{\prime} 18^{\prime \prime} \mathrm{N}$ & $23^{\circ} 14^{\prime} 41^{\prime \prime} \mathrm{E}$ & 14.09 .2000 & T. Ljubomirov & Non-metallic \\
\hline 우 & Bulgaria, north of Tchuypetlovo & $42^{\circ} 31^{\prime} 18^{\prime \prime} \mathrm{N}$ & $23^{\circ} 14^{\prime} 41^{\prime \prime} \mathrm{E}$ & 14.09 .2000 & T. Ljubomirov & Non-metallic \\
\hline 우 & Bulgaria, north of Tchuypetlovo & $42^{\circ} 31^{\prime} 18^{\prime \prime} \mathrm{N}$ & $23^{\circ} 14^{\prime} 41^{\prime \prime} \mathrm{E}$ & 14.09 .2000 & T. Ljubomirov & Non-metallic \\
\hline 우 & Bulgaria, south of Kladnitsa & $42^{\circ} 32^{\prime} 27^{\prime \prime} \mathrm{N}$ & $23^{\circ} 11^{\prime} 22^{\prime \prime} \mathrm{E}$ & 1.09 .1998 & T. Ljubomirov & Non-metallic \\
\hline 우 & Bulgaria, south of Kladnitsa & $42^{\circ} 32^{\prime} 27^{\prime \prime} \mathrm{N}$ & $23^{\circ} 11^{\prime} 22^{\prime \prime} \mathrm{E}$ & 1.09 .1998 & T. Ljubomirov & Non-metallic \\
\hline 우 & Bulgaria, south-east of Kladnitsa & $42^{\circ} 32^{\prime} 06^{\prime \prime} \mathrm{N}$ & $23^{\circ} 12^{\prime} 42^{\prime \prime} \mathrm{E}$ & 25.08 .2000 & T. Ljubomirov & Non-metallic \\
\hline 우 & Bulgaria, south of Sofia & $42^{\circ} 35^{\prime} 54^{\prime \prime} \mathrm{N}$ & $23^{\circ} 14^{\prime} 49^{\prime \prime} \mathrm{E}$ & 22.08 .2000 & T. Ljubomirov & Non-metallic \\
\hline 우 & Bulgaria, south of Sofia & $42^{\circ} 35^{\prime} 54^{\prime \prime} \mathrm{N}$ & $23^{\circ} 14^{\prime} 49^{\prime \prime} \mathrm{E}$ & 22.08 .2000 & T. Ljubomirov & Non-metallic \\
\hline 우 & Bulgaria, south-west of Gorna Breznitsa & $41^{\circ} 43^{\prime} 12^{\prime \prime} \mathrm{N}$ & $23^{\circ} 06^{\prime} 39^{\prime \prime} \mathrm{E}$ & 19.06 .2003 & T. Ljubomirov & Non-metallic \\
\hline 우 & Bulgaria, south-west of Mikrevo & $41^{\circ} 36^{\prime} 21^{\prime \prime} \mathrm{N}$ & $23^{\circ} 09^{\prime} 07^{\prime \prime} \mathrm{E}$ & 27.06 .2008 & T. Ljubomirov & Non-metallic \\
\hline 우 & Bulgaria, west of Deltchevo & $41^{\circ} 33^{\prime} 11^{\prime \prime} \mathrm{N}$ & $23^{\circ} 38^{\prime} 00^{\prime \prime} \mathrm{E}$ & 28.04 .2004 & O. Todorov & Non-metallic \\
\hline q & Bulgaria, west of Etropole & $42^{\circ} 49^{\prime} 55^{\prime \prime} \mathrm{N}$ & $23^{\circ} 57^{\prime} 44^{\prime \prime} \mathrm{E}$ & 1.07 .2010 & T. Ljubomirov & Non-metallic \\
\hline$\hat{\delta}$ & Bulgaria, west of Etropole & $42^{\circ} 49^{\prime} 55^{\prime \prime} \mathrm{N}$ & $23^{\circ} 57^{\prime} 44^{\prime \prime} \mathrm{E}$ & 1.07.2010 & T. Ljubomirov & Metallic red \\
\hline P & Bulgaria, west of Etropole & $42^{\circ} 50^{\prime} 30^{\prime \prime} \mathrm{N}$ & $23^{\circ} 58^{\prime} 17^{\prime \prime} \mathrm{E}$ & 13.08 .2018 & T. Ljubomirov & Non-metallic \\
\hline 9 & Turkey, south-west of Eyüpler & $37^{\circ} 44^{\prime} 58^{\prime \prime} \mathrm{N}$ & $30^{\circ} 49^{\prime} 18^{\prime \prime} \mathrm{E}$ & 29.07 .2008 & T. Ljubomirov & Non-metallic \\
\hline $\bar{\delta}$ & Italy, Vicarello & $43^{\circ} 37^{\prime} 20^{\prime \prime} \mathrm{N}$ & $10^{\circ} 26^{\prime} 40^{\prime \prime} \mathrm{E}$ & 15.09 .1991 & F. Strumia & Non-metallic \\
\hline q & Italy, Chiani & $43^{\circ} 27^{\prime} 50^{\prime \prime} \mathrm{N}$ & $10^{\circ} 40^{\prime} 44^{\prime \prime} \mathrm{E}$ & $29.07-09.08 .2001$ & F. Strumia & Non-metallic \\
\hline 9 & Italy, Chiani & $43^{\circ} 27^{\prime} 50^{\prime \prime} \mathrm{N}$ & $10^{\circ} 40^{\prime} 44^{\prime \prime} \mathrm{E}$ & $11-16.08 .2000$ & F. Strumia & Non-metallic \\
\hline q & Italy, Piombino & $42^{\circ} 56^{\prime} 44^{\prime \prime} \mathrm{N}$ & $10^{\circ} 29^{\prime} 56^{\prime \prime} \mathrm{E}$ & $4-18.07 .2000$ & F. Strumia & Non-metallic \\
\hline
\end{tabular}




\begin{tabular}{|c|c|c|c|c|c|c|}
\hline q & Italy, Vicchio, Firenze & $43^{\circ} 33^{\prime} 20^{\prime \prime} \mathrm{N}$ & $10^{\circ} 32^{\prime} 53^{\prime \prime} \mathrm{E}$ & 6-16.09.1998 & F. Strumia & Non-metallic \\
\hline q & Italy, Vicchio, Firenze & $43^{\circ} 33^{\prime} 20^{\prime \prime} \mathrm{N}$ & $10^{\circ} 32^{\prime} 53^{\prime \prime} \mathrm{E}$ & $25-30.07$ & F. Strumia & Non-metallic \\
\hline q & Italy, Vicchio, Firenze & $43^{\circ} 33^{\prime} 20^{\prime \prime} \mathrm{N}$ & $10^{\circ} 32^{\prime} 53^{\prime \prime} \mathrm{E}$ & $27.08-06.09 .1998$ & F. Strumia & Non-metallic \\
\hline q & Italy, Vicchio, Firenze & $43^{\circ} 33^{\prime} 20^{\prime \prime} \mathrm{N}$ & $10^{\circ} 32^{\prime} 53^{\prime \prime} \mathrm{E}$ & $11-26.061998$ & F. Strumia & Non-metallic \\
\hline P & Italy, Vicchio, Firenze & $43^{\circ} 33^{\prime} 20^{\prime \prime} \mathrm{N}$ & $10^{\circ} 32^{\prime} 53^{\prime \prime} \mathrm{E}$ & $27.06-05.07 .1998$ & F. Strumia & Non-metallic \\
\hline 9 & Italy, Vicchio, Firenze & $43^{\circ} 33^{\prime} 20^{\prime \prime} \mathrm{N}$ & $10^{\circ} 32^{\prime} 53^{\prime \prime} \mathrm{E}$ & 18.08 .1991 & F. Strumia & Non-metallic \\
\hline$q$ & Italy, Caselli Nat. reserve Pisa & $43^{\circ} 13^{\prime} 46^{\prime \prime} \mathrm{N}$ & $10^{\circ} 41^{\prime} 57^{\prime \prime} \mathrm{E}$ & $20.07-02.08$ & F. Strumia & Non-metallic \\
\hline q & Italy, Gabbro & $43^{\circ} 28^{\prime} 45^{\prime \prime} \mathrm{N}$ & $10^{\circ} 26^{\prime} 37^{\prime \prime} \mathrm{E}$ & $29.08-06.09 .2014$ & F. Strumia & Non-metallic \\
\hline$\hat{0}$ & Italy, Caselli Nat. reserve Pisa & $43^{\circ} 13^{\prime} 46^{\prime \prime} \mathrm{N}$ & $10^{\circ} 41^{\prime} 57^{\prime \prime} \mathrm{E}$ & $20.06-02.07 .2019$ & F. Strumia & Metallic red \\
\hline$\hat{0}$ & Italy, Pisa & $43^{\circ} 40^{\prime} 31^{\prime \prime} \mathrm{N}$ & $19^{\circ} 19^{\prime} 36^{\prime \prime} \mathrm{E}$ & 19.09 .1991 & F. Strumia & Non-metallic \\
\hline$\hat{\sigma}$ & Italy, Marano, Pistoia & $45^{\circ} 44^{\prime} 28^{\prime \prime} \mathrm{N}$ & $13^{\circ} 08^{\prime} 25^{\prime \prime} \mathrm{E}$ & 17.07 .1989 & F. Strumia & Non-metallic \\
\hline q & Italy, Cengio, Savona & $44^{\circ} 23^{\prime} 10^{\prime \prime} \mathrm{N}$ & $8^{\circ} 11^{\prime} 36^{\prime \prime} \mathrm{E}$ & 4.09 .1948 & L. Berio & Non-metallic \\
\hline P & Italy, Cengio, Savona & $44^{\circ} 23^{\prime} 10^{\prime \prime} \mathrm{N}$ & $8^{\circ} 11^{\prime} 36^{\prime \prime} \mathrm{E}$ & 4.09 .1948 & L. Berio & Non-metallic \\
\hline q & Italy, Chianni, Pisa & $43^{\circ} 27^{\prime} 50^{\prime \prime} \mathrm{N}$ & $10^{\circ} 40^{\prime} 44^{\prime \prime} \mathrm{E}$ & $3-11.08 .2000$ & F. Strumia & Non-metallic \\
\hline$q$ & France, Corsica, Castirla & $42^{\circ} 22^{\prime} 58^{\prime \prime} \mathrm{N}$ & $9^{\circ} 09^{\prime} 08^{\prime \prime} \mathrm{E}$ & $24.06-24.07 .2000$ & F. Strumia & Non-metallic \\
\hline q & France, Corsica, Castirla & $42^{\circ} 22^{\prime} 58^{\prime \prime} \mathrm{N}$ & $9^{\circ} 09^{\prime} 08^{\prime \prime} \mathrm{E}$ & $27.06-08.07 .2001$ & F. Strumia & Non-metallic \\
\hline 9 & France, Corsica, Castirla & $42^{\circ} 22^{\prime} 58^{\prime \prime} \mathrm{N}$ & $9^{\circ} 09^{\prime} 08^{\prime \prime} \mathrm{E}$ & $3-15.07 .2002$ & F. Strumia & Non-metallic \\
\hline$\hat{0}$ & France, Corsica, Castirla & $42^{\circ} 22^{\prime} 58^{\prime \prime} \mathrm{N}$ & $9^{\circ} 09^{\prime} 08^{\prime \prime} \mathrm{E}$ & 30.06-13.07.1999 & F. Strumia & Non-metallic \\
\hline$\hat{\sigma}$ & France, Corsica, Castirla & $42^{\circ} 22^{\prime} 58^{\prime \prime} \mathrm{N}$ & $9^{\circ} 09^{\prime} 08^{\prime \prime} \mathrm{E}$ & $1-23.08 .2002$ & F. Strumia & Non-metallic \\
\hline q & France, Corsica, Castirla & $42^{\circ} 22^{\prime} 58^{\prime \prime} \mathrm{N}$ & $9^{\circ} 09^{\prime} 08^{\prime \prime} \mathrm{E}$ & $3-15.07 .2002$ & F. Strumia & Non-metallic \\
\hline q & France, Corsica, Castirla & $42^{\circ} 22^{\prime} 58^{\prime \prime} \mathrm{N}$ & $9^{\circ} 09^{\prime} 08^{\prime \prime} \mathrm{E}$ & 30.06-16.07.1999 & F. Strumia & Non-metallic \\
\hline q & France, Corsica, Cuttoli & $41^{\circ} 57^{\prime} 50^{\prime \prime} \mathrm{N}$ & $8^{\circ} 50^{\prime} 50^{\prime \prime} \mathrm{E}$ & $10-25.07 .2002$ & F. Strumia & Non-metallic \\
\hline$\hat{\sigma}$ & France, Corsica, Cargese & $42^{\circ} 08^{\prime} 10^{\prime \prime} \mathrm{N}$ & $8^{\circ} 35^{\prime} 30^{\prime \prime} \mathrm{E}$ & $21-22.08 .1998$ & F. Strumia & Metallic red \\
\hline q & France, Corsica, Cargese & $42^{\circ} 08^{\prime} 10^{\prime \prime} \mathrm{N}$ & $8^{\circ} 35^{\prime} 30^{\prime \prime} \mathrm{E}$ & $21-22.08 .1998$ & F. Strumia & Non-metallic \\
\hline 우 & France, Corsica, Asco & $42^{\circ} 28^{\prime} 30^{\prime \prime} \mathrm{N}$ & $9^{\circ} 01^{\prime} 20^{\prime \prime} \mathrm{E}$ & 4.07 .2002 & F. Strumia & Non-metallic \\
\hline$\hat{\sigma}^{\pi}$ & France, Corsica, Ponte Leccia & $42^{\circ} 27^{\prime} 07^{\prime \prime} \mathrm{N}$ & $9^{\circ} 14^{\prime} 40^{\prime \prime} \mathrm{E}$ & 14.06 .1997 & F. Strumia & Non-metallic \\
\hline 우 & France, Corsica, Ponte Leccia & $42^{\circ} 27^{\prime} 07^{\prime \prime} \mathrm{N}$ & $9^{\circ} 14^{\prime} 40^{\prime \prime} \mathrm{E}$ & 14.06 .1997 & F. Strumia & Non-metallic \\
\hline 우 & France, Corsica, Ponte Leccia & $42^{\circ} 27^{\prime} 07^{\prime \prime} \mathrm{N}$ & $9^{\circ} 14^{\prime} 40^{\prime \prime} \mathrm{E}$ & 14.06 .1997 & F. Strumia & Non-metallic \\
\hline Q & France, Corsica, Ponte Leccia & $42^{\circ} 27^{\prime} 07^{\prime \prime} \mathrm{N}$ & $9^{\circ} 14^{\prime} 40^{\prime \prime} \mathrm{E}$ & 14.06 .1997 & F. Strumia & Non-metallic \\
\hline 우 & France, Corsica, Ponte Leccia & $42^{\circ} 27^{\prime} 07^{\prime \prime} \mathrm{N}$ & $9^{\circ} 14^{\prime} 40^{\prime \prime} \mathrm{E}$ & 14.06 .1997 & F. Strumia & Non-metallic \\
\hline 우 & France, Corsica, Ponte Leccia & $42^{\circ} 27^{\prime} 07^{\prime \prime} \mathrm{N}$ & $9^{\circ} 14^{\prime} 40^{\prime \prime} \mathrm{E}$ & 14.06 .1997 & F. Strumia & Non-metallic \\
\hline q & France, Corsica, Ponte Leccia & $42^{\circ} 27^{\prime} 07^{\prime \prime} \mathrm{N}$ & $9^{\circ} 14^{\prime} 40^{\prime \prime} \mathrm{E}$ & 14.06 .1997 & F. Strumia & Non-metallic \\
\hline 우 & France, Corsica, Ponte Leccia & $42^{\circ} 27^{\prime} 07^{\prime \prime} \mathrm{N}$ & $9^{\circ} 14^{\prime} 40^{\prime \prime} \mathrm{E}$ & 14.06 .1997 & F. Strumia & Non-metallic \\
\hline$\hat{0}$ & France, Corsica, La Marana & $42^{\circ} 34^{\prime} 01^{\prime \prime} \mathrm{N}$ & $9^{0} 31^{\prime} 32^{\prime \prime} \mathrm{E}$ & 14.06 .1998 & F. Strumia & Non-metallic \\
\hline q & France, Corsica, La Marana & $42^{\circ} 34^{\prime} 01^{\prime \prime} \mathrm{N}$ & $9^{\circ} 31^{\prime} 32^{\prime \prime} \mathrm{E}$ & $18-26.08 .1999$ & F. Strumia & Non-metallic \\
\hline$\hat{0}$ & France, Corsica, La Marana & $42^{\circ} 34^{\prime} 01^{\prime \prime} \mathrm{N}$ & $9^{\circ} 31^{\prime} 32^{\prime \prime} \mathrm{E}$ & 7.-10.07.1998 & F. Strumia & Non-metallic \\
\hline$\hat{0}$ & France, Corsica, La Marana & $42^{\circ} 34^{\prime} 01^{\prime \prime} \mathrm{N}$ & $9^{\circ} 31^{\prime} 32^{\prime \prime} \mathrm{E}$ & 7.-10.07.1998 & F. Strumia & Non-metallic \\
\hline$\hat{0}$ & France, Corsica, La Marana & $42^{\circ} 34^{\prime} 01^{\prime \prime} \mathrm{N}$ & $9^{0} 31^{\prime} 32^{\prime \prime} \mathrm{E}$ & $21-22.08 .1998$ & F. Strumia & Non-metallic \\
\hline 우 & France, Corsica, Conca Separellu & $41^{\circ} 43^{\prime} 50^{\prime \prime} \mathrm{N}$ & $9^{\circ} 20^{\prime} 10^{\prime \prime} \mathrm{E}$ & 7.08 .2004 & F. Strumia & Non-metallic \\
\hline$\hat{0}$ & France, Corsica, Pielza & $41^{\circ} 54^{\prime} 11^{\prime \prime} \mathrm{N}$ & $9^{\circ} 23^{\prime} 30^{\prime \prime} \mathrm{E}$ & 13.06 .1997 & F. Strumia & Metallic red \\
\hline
\end{tabular}

Table 3. Data on the studied individuals of Hedychridium rossicum. 


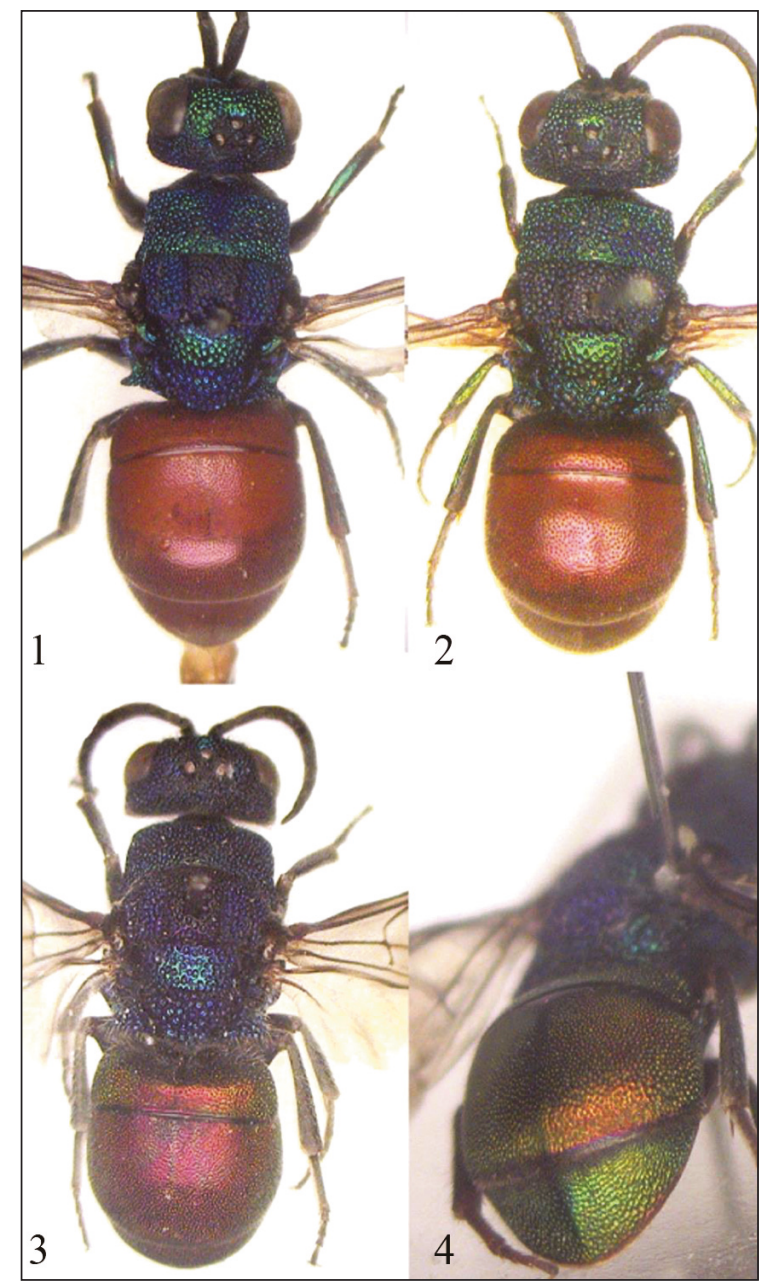

Figures 1-4. Hedychridium rossicum Gussakovskij, 1948. Fig. 1: female with metasoma non-metallic (Bulgaria, south of Milanovo village, $764 \mathrm{~m}, 07.07 .2015)$. Fig. 2: male with metasoma non-metallic (Bulgaria, north-west of Barutin village, $112 \mathrm{~m}, 07.08 .2018)$. Fig. 3: male with metasoma metallic red (Bulgaria, west of Zheleznitsa village, $1140 \mathrm{~m}, 14.07 .1999)$. Fig. 4: male with metasoma bicolor: second tergum metallic red, third tergum metallic green (Bulgaria, north-west of Bisstritsa village, $1100 \mathrm{~m}$, 05.08.1998).

\section{Hedychridium insulare Balthasar, 1953}

= Hedychridium roseum var. cypriacum Balthasar, 1953

= Hedychridium lampadum limassolense Linsenmaier, 1959

= Hedychridium mediocrum Linsenmaier, 1987

$=$ Hedychridium mediocrate Kimsey et R. Bohart, 1990

Distribution. Southwest Europe, Hungary,
Ukraine, Near East, Cyprus, Syria, Armenia, Kazakhstan (Arens, 2010a).

REMARKS ON COLORATION. Only four females and two males were captured. All females have non-metallic metasoma while the two males have a metallic red metasoma. The study of more males is needed to establish the possible dimorphism in metasoma color. From Syria, Cyprus, Turkey and Greece we could study additional 10 females of $H$. insulare: all with non-metallic metasoma.

\section{Hedychridium roseum (Rossi, 1790)}

= Hedychridium lampadum Linsenmaier, 1959

= Hedychridium lampadum sorianum Linsenmaier, 1959

= Hedychridium lampadum anatolicum Arens, 2010

Distribution. Europe, Morocco, East Asia, Ukraine, Bulgaria, Greece Manchuria, Siberia, Russia (Martynova, 2015).

REMARKS ON COLORATION. In Bulgaria 6 males and 11 females were captured. All females show a non-metallic metasoma, while two males show metallic red metasoma coloration.

\section{DISCUSSION}

Tables 1-6 resume our captures from Hedychridium roseum species-group of Bulgaria. From this data follow a complex situation for the metasoma metallic color: this feature is unstable and variable. Apparently, the metasoma color suggests a connection to a sexual chromosome. The distance between reflecting layers in cuticle seems to be genetically variable. Fortunately, the identification keys of Arens are still useful, provided to skip metasoma color feature. The number of studied individuals from Bulgaria is limited (118) and for some uncommon species it is even too low to have a significant statistical meaning (Table 7).

We have found that in the majority of females in $H$. roseum species-group the metasoma is nonmetallic. Only in $H$. roseum a few Bulgarian females have the metasoma metallic red. In males the metasoma coloration is more variable and unpredictable. Arens (2010a) considered H. sculpturatum 


\begin{tabular}{|c|c|c|c|c|c|c|}
\hline Sex & Locality & Latitude & Longitude & Date & Collector & \begin{tabular}{|l|} 
Metasoma \\
color
\end{tabular} \\
\hline$\hat{\sigma}$ & Bulgaria, north-west of Passarel & $42^{\circ} 33^{\prime} 12^{\prime \prime} \mathrm{N}$ & $23^{\circ} 29^{\prime} 34^{\prime \prime} \mathrm{E}$ & 4.07.2013 & T. Ljubomirov & Metallic red \\
\hline q & Bulgaria, north-west of Passarel & $42^{\circ} 33^{\prime} 05^{\prime \prime} \mathrm{N}$ & $23^{\circ} 29^{\prime} 30^{\prime \prime} \mathrm{E}$ & 4.07 .2013 & T. Ljubomirov & Non-metallic \\
\hline q & Bulgaria, north-west of Passarel & $42^{\circ} 33^{\prime} 05^{\prime \prime} \mathrm{N}$ & $23^{\circ} 29^{\prime} 30^{\prime \prime} \mathrm{E}$ & 29.07 .2001 & T. Ljubomirov & Non-metallic \\
\hline 우 & Bulgaria, north-west of Passarel & $42^{\circ} 33^{\prime} 05^{\prime \prime} \mathrm{N}$ & $23^{\circ} 29^{\prime} 30^{\prime \prime} \mathrm{E}$ & 24.07 .2002 & T. Ljubomirov & Non-metallic \\
\hline 우 & Bulgaria, north-west of Passarel & $42^{\circ} 33^{\prime} 05^{\prime \prime} \mathrm{N}$ & $23^{\circ} 29^{\prime} 30^{\prime \prime} \mathrm{E}$ & 24.07 .2002 & T. Ljubomirov & Non-metallic \\
\hline 우 & Bulgaria, north-west of Passarel & $42^{\circ} 33^{\prime} 05^{\prime \prime} \mathrm{N}$ & $23^{\circ} 29^{\prime} 30^{\prime \prime} \mathrm{E}$ & 24.07 .2002 & T.Ljubomirov & Non-metallic \\
\hline q & Bulgaria, south-west of Gorna Breznitsa & $41^{\circ} 44^{\prime} 38^{\prime \prime} \mathrm{N}$ & $23^{\circ} 06^{\prime} 14^{\prime \prime} \mathrm{E}$ & 16.06 .2002 & T. Ljubomirov & Non-metallic \\
\hline q & Bulgaria, Svilengrad & $41^{\circ} 46^{\prime} 28^{\prime \prime} \mathrm{N}$ & $26^{\circ} 08^{\prime} 36^{\prime \prime} \mathrm{E}$ & 19.05 .2010 & T. Ljubomirov & Non-metallic \\
\hline q & Bulgaria, north of Enina & $42^{\circ} 40^{\prime} 50^{\prime \prime} \mathrm{N}$ & $25^{\circ} 24^{\prime} 57^{\prime \prime} \mathrm{E}$ & $23-29.07 .2000$ & M. Langourov & Non-metallic \\
\hline q & Bulgaria, north of Passarel & $42^{\circ} 33^{\prime} 54^{\prime \prime} \mathrm{N}$ & $23^{\circ} 29^{\prime} 30^{\prime \prime} \mathrm{E}$ & 29.07 .2001 & T. Ljubomirov & Non-metallic \\
\hline q & Bulgaria, south-west of Zemen & $42^{\circ} 28^{\prime} 19^{\prime \prime} \mathrm{N}$ & $22^{\circ} 44^{\prime} 06^{\prime \prime} \mathrm{E}$ & 13.07 .2016 & T. Ljubomirov & Non-metallic \\
\hline q & Turkey, north of Küllü & $36^{\circ} 56^{\prime} 04^{\prime \prime} \mathrm{N}$ & $36^{\circ} 24^{\prime} 02^{\prime \prime} \mathrm{E}$ & $14-21.07 .2007$ & M.F. Gürbüz & Non-metallic \\
\hline
\end{tabular}

Table 4. Data on the studied individuals of Hedychridium caputaureum.

\begin{tabular}{|c|c|c|c|c|c|c|}
\hline $\operatorname{Sex}$ & Locality & Latitude & Longitude & Date & Collector & $\begin{array}{l}\text { Metasoma } \\
\text { color }\end{array}$ \\
\hline$\hat{0}$ & Bulgaria, north of Yakovo & $41^{\circ} 31^{\prime} 21^{\prime \prime} \mathrm{N}$ & $23^{\circ} 07^{\prime} 50^{\prime \prime} \mathrm{E}$ & 3.07 .2012 & T. Ljubomirov & Metallic red \\
\hline $0^{\pi}$ & Bulgaria, south of Klokotnita & $41^{\circ} 58^{\prime} 31^{\prime \prime} \mathrm{N}$ & $25^{\circ} 29^{\prime} 39^{\prime \prime} \mathrm{E}$ & 21.07 .2005 & T. Ljubomirov & Metallic red \\
\hline 우 & Bulgaria, south-west of Gorna Breznitsa & $41^{\circ} 43^{\prime} 12^{\prime \prime} \mathrm{N}$ & $23^{\circ} 06^{\prime} 39^{\prime \prime} \mathrm{E}$ & 15.07 .2003 & T. Ljubomirov & Non-metallic \\
\hline q & Bulgaria, south-west of Gorna Breznitsa & $41^{\circ} 43^{\prime} 12^{\prime \prime} \mathrm{N}$ & $23^{\circ} 06^{\prime} 39^{\prime \prime} \mathrm{E}$ & 15.07 .2003 & T. Ljubomirov & Non-metallic \\
\hline 우 & Turkey, Mitisin & $36^{\circ} 59^{\prime} 02^{\prime \prime} \mathrm{N}$ & $36^{\circ} 22^{\prime} 29^{\prime \prime} \mathrm{E}$ & 22.07 .2008 & T. Ljubomirov & Non-metallic \\
\hline q & Turkey, Gökçaay suburb & $37^{\circ} 44^{\prime} 52^{\prime \prime} \mathrm{N}$ & $30^{\circ} 32^{\prime} 28^{\prime \prime} \mathrm{E}$ & 29.07 .2004 & J. Kolarov & Non-metallic \\
\hline q & Cyprus, Limassol & - & - & 1.06 .1949 & A. Mochi & Non-metallic \\
\hline 우 & Cyprus, Limassol & - & - & 3.06 .1949 & A. Mochi & Non-metallic \\
\hline q & Syria, Damascus & - & - & 11.10 .1954 & A. Mochi & Non-metallic \\
\hline q & Syria, Damascus & - & - & 10.10 .1952 & A. Mochi & Non-metallic \\
\hline q & Greece, Kiparissia & - & - & 19.06.1955 & G. Pagliano & Non-metallic \\
\hline$q$ & Greece, Kiparissia & - & - & 19.06 .1955 & G. Pagliano & Non-metallic \\
\hline q & Turkey, Erzurum & - & - & 12.08 .1992 & E. Yildirim & Non-metallic \\
\hline
\end{tabular}

Table 5. Data on the studied individuals of Hedychridium insulare.

a sexual dimorphic species with females' nonmetallic and male's metallic red metasoma. The data in Table 3 confirm this statement even though it is based on a single available male. For $\mathrm{H}$. scutellare we find all females non-metallic while Arens (2010a) assume that the metasoma both in the males and the females is all red. In $H$. caputaureum the single male is metallic red and the Arens' (2010a) sentence is in agreement with our data. The six individuals of $H$. insulare are in agreement with Arens' statement.

In $H$. roseum the majority of individuals (both males and females) exhibit non-metallic metasoma but among the six males studied two have metallic red metasoma ( $12 \%$ of available data) thus evidencing the need of additional investigation. 


\begin{tabular}{|c|c|c|c|c|c|c|}
\hline Sex & Locality & Latitude & Longitude & Date & Collector & $\begin{array}{c}\text { Metasoma } \\
\text { color }\end{array}$ \\
\hline$\hat{\sigma}$ & Bulgaria, north-west of Passarel & $42^{\circ} 33^{\prime} 05^{\prime \prime} \mathrm{N}$ & $23^{\circ} 29^{\prime} 30^{\prime \prime} \mathrm{E}$ & 21.06 .1997 & T. Ljubomirov & Non-metallic \\
\hline 0 & Bulgaria, west of Milanovo & $43^{\circ} 05^{\prime} 40^{\prime \prime} \mathrm{N}$ & $23^{\circ} 23^{\prime} 23^{\prime \prime} \mathrm{E}$ & 7.07.2015 & T. Ljubomirov & Metallic red \\
\hline 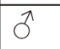 & Bulgaria, south of Petrich & $41^{\circ} 22^{\prime} 05^{\prime \prime} \mathrm{N}$ & $23^{\circ} 11^{\prime} 14^{\prime \prime} \mathrm{E}$ & $10-20.07 .2002$ & O. Todorov & Metallic red \\
\hline ô & Bulgaria, south-east of Kladnitsa & $42^{\circ} 32^{\prime} 49^{\prime \prime} \mathrm{N}$ & $23^{\circ} 12^{\prime} 07^{\prime \prime} \mathrm{E}$ & 25.08 .2000 & T. Ljubomirov & Red metallic \\
\hline P & Bulgar & $2^{\prime \prime N}$ & $6^{\prime \prime} \mathrm{E}$ & 31.08 .2015 & T. Lj & Non \\
\hline$\hat{0}$ & Bulgaria, west of Dobrostan & $41^{\circ} 53^{\prime} 22^{\prime \prime} \mathrm{N}$ & $24^{\circ} 52^{\prime} 54^{\prime \prime} \mathrm{E}$ & 24.08 .2013 & T. Ljubomirov & Non-metallic \\
\hline q & Bulgaria, Banitsa & $43^{\circ} 20^{\prime} 20^{\prime \prime} \mathrm{N}$ & $23^{\circ} 41^{\prime} 07^{\prime \prime} \mathrm{E}$ & $16-30.06 .2013$ & L. Toshkov & Non-metallic \\
\hline P & Bulgaria, south of Sofia & $42^{\circ} 38^{\prime} 10^{\prime \prime} \mathrm{N}$ & $23^{\circ} 14^{\prime} 38^{\prime \prime} \mathrm{E}$ & 14.08 .2000 & T. Ljubomirov & Non-metallic \\
\hline q & Bulgaria, east of Borino & $41^{\circ} 40^{\prime} 37^{\prime \prime} \mathrm{N}$ & $24^{\circ} 19^{\prime} 22^{\prime \prime} \mathrm{E}$ & 16.08 .2016 & T. Ljubomirov & Non-metallic \\
\hline q & Bulgaria, east of Kladnitsa & $42^{\circ} 33^{\prime} 29^{\prime \prime} \mathrm{N}$ & $23^{\circ} 12^{\prime} 12^{\prime \prime} \mathrm{E}$ & 25.08 .2000 & T. Ljubomirov & Non-metallic \\
\hline q & Bulgaria, west of Zheleznitsa & $42^{\circ} 32^{\prime} 08^{\prime \prime} \mathrm{N}$ & $23^{\circ} 20^{\prime} 44^{\prime \prime} \mathrm{E}$ & 28.08 .2000 & T. Ljubomirov & Non-metallic \\
\hline 우 & Bulgaria, west of Zheleznitsa & $42^{\circ} 32^{\prime} 13^{\prime \prime} \mathrm{N}$ & $23^{\circ} 20^{\prime} 24^{\prime \prime} \mathrm{E}$ & 6.08 .1998 & T. Ljubomirov & Non-metallic \\
\hline 우 & Bulgaria, north of Tchuypetlovo & $42^{\circ} 31^{\prime} 18^{\prime \prime} \mathrm{N}$ & $23^{\circ} 14^{\prime} 41^{\prime \prime} \mathrm{E}$ & 14.09 .2000 & T. Ljubomirov & Non-metallic \\
\hline 우 & Bulgaria, east of Bossnek & $42^{\circ} 29^{\prime} 03^{\prime \prime} \mathrm{N}$ & $23^{\circ} 13^{\prime} 38^{\prime \prime} \mathrm{E}$ & 19.08 .1998 & T. Ljubomirov & Non-metallic \\
\hline 우 & Bulgaria, Batchkovo & $41^{\circ} 55^{\prime} 59^{\prime \prime} \mathrm{N}$ & $24^{\circ} 51^{\prime} 58^{\prime \prime} \mathrm{E}$ & 9.08 .2013 & T. Ljubomirov & Non-metallic \\
\hline P & Bulgaria, south-west of Zheleznitsa & $42^{\circ} 31^{\prime} 35^{\prime \prime} \mathrm{N}$ & $23^{\circ} 21^{\prime} 06^{\prime \prime} \mathrm{E}$ & 23.07 .1998 & T. Ljubomirov & Non-metallic \\
\hline q & Bulgaria, west of Tipchenitsa & $43^{\circ} 03^{\prime} 56^{\prime \prime} \mathrm{N}$ & $23^{\circ} 41^{\prime} 42^{\prime \prime} \mathrm{E}$ & 7.06 .2009 & T. Ljubomirov & Non-metallic \\
\hline
\end{tabular}

Table 6. Data on the studied individuals of Hedychridium roseum.

\begin{tabular}{|l|c|}
\hline $\begin{array}{c}\text { Studied individuals of Hedychridium roseum species-group } \\
\text { from Bulgaria }\end{array}$ & \\
\hline Hedychridium insulare Balthasar, 1953 & 4 \\
\hline Hedychridium scutellare (Tournier, 1878) & 5 \\
\hline H. sculpturatum (Abeille de Perrin, 1877) & 9 \\
\hline Hedychridium caputaureum Trautmann \&Traut., 1919 & 11 \\
\hline Hedychridium roseum (Rossi, 1790) & 17 \\
\hline Hedychridium rossicum Gussakovskij, 1948 & 72 \\
\hline
\end{tabular}

Table 7. Studied individuals of Hedychridium roseum species-group from Bulgaria.

In $H$. rossicum we found that only $75 \%$ of males have metallic red metasoma; further, two individuals exhibit a bicolor metallic red and green metasoma; all females are non-metallic. Hedychridium rossicum is the most frequent species both in Bulgaria and in Italy. It is also the most variable regarding the metasoma coloration in males and females, as shown in the Table 4.

Our results from Bulgaria are in agreement with the studied material from Tuscany, Sardinia, and Corsica.

\section{CONCLUSIONS}

Our results confirm the presence in the Hedychridium roseum species-group of either individual with or without interference color on Metasoma. It should be expected that the evolutionary pressure must select the most favorable color cancelling the less favorable. If this do not happens some special effect on biology can preserve such a dimorphic population. A careful study of individuals' biology would suggest a possible explanation. Unfortunately Chrysididae are uncommon, fast moving and difficult to observe in the field. Some statistical insight can be searched and tested if enough material from a given locality becomes available.

We have data for 15 Hedychridium roseum individuals captured in Tuscany around Pisa.

In Fig. 5 the time distribution of captures is shown. Apparently the two color forms are active at different summer times, thus with a different in- 


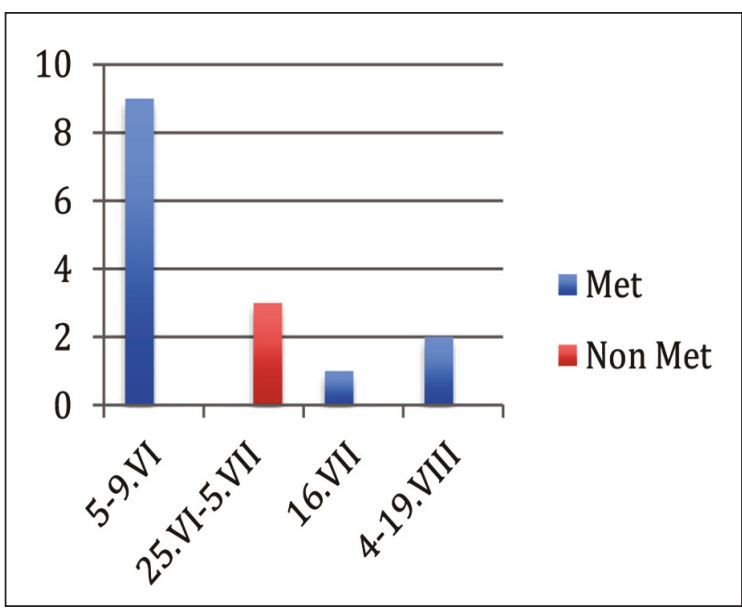

Figure 5. Time distribution of $H$. rossicum captures during the year.

teraction with the surrounding places and a different selection pressure. This is a possible explanation, even if the too reduced statistic can only suggest a direction for future investigations. The red metallic individuals are apparently more present in spring and in July and August. On the contrary, the less frequent individuals with non-metallic Metasoma were captured between June and July. The relative abundance of the six species captured in Bulgaria is summarized in Fig. 6.

The prevalence of $H$. rossicum may reflects a preference of collectors for locations most favor-

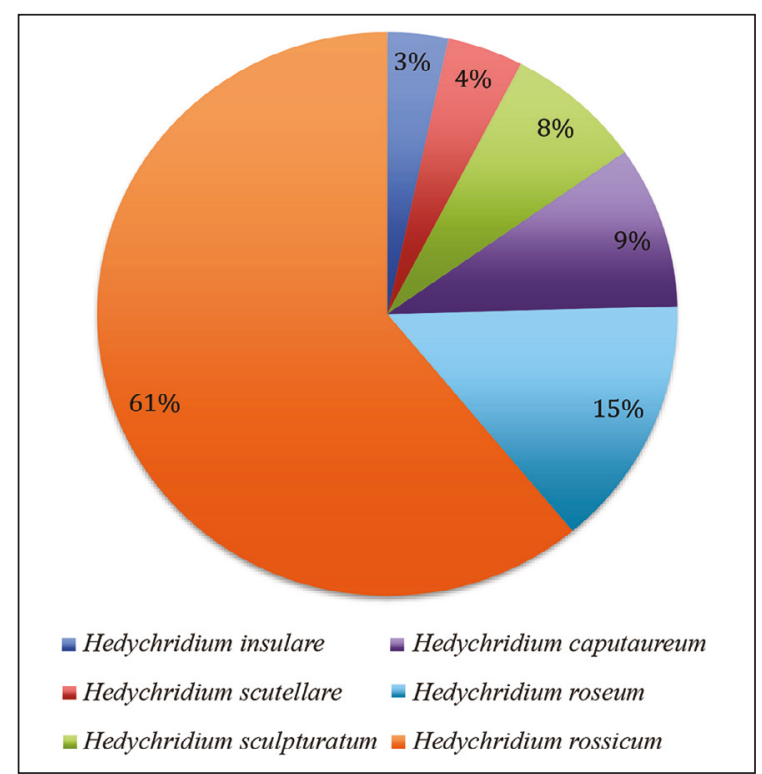

Figure 6. Relative abundance of the Hedychridium roseum species-group as observed in the Bulgarian material.

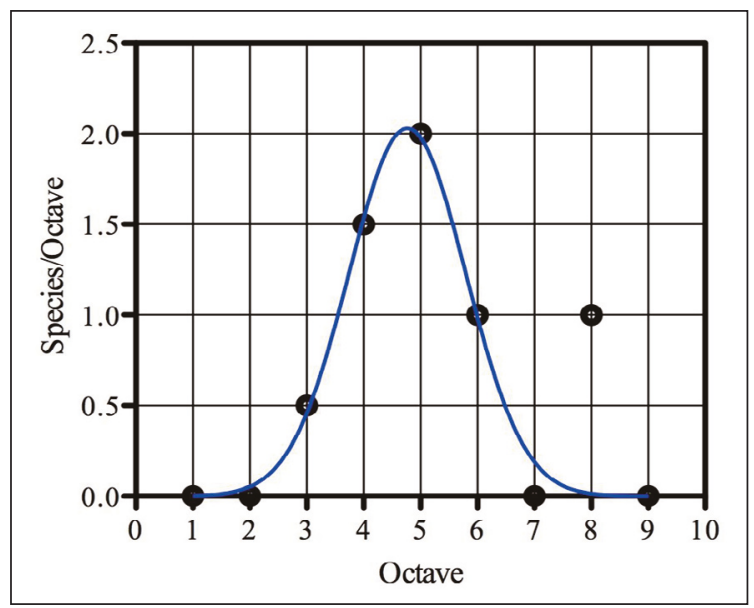

Figure 7. Lognormal distribution of the H. roseum- group species captured in Bulgaria. Note that the Lognormal is entirely restricted to positive values of Octaves.

able to this species. The lognormal distribution can be calculated from above data and the result is shown in Fig. 7. Notably the lognormal distribution is entirely restricted to positive values of octaves. This shows that the presence of further species is not needed to optimize the lognormal distribution. Thus it follows that the observed six species of the Hedychridium roseum-group are the only one present in Bulgaria, and. no further species should be captured by further investigations.

In conclusion, the two-color form appears to be active in different time intervals and thus subjected to possible, different or independent, evolutionary selection pressure. Unfortunately the data numbers are too small and only a possible explanation can be suggested.

\section{REFERENCES}

Arens W., 2004. Beitrag zur Taxonomie griechischer Goldwespen, mit Beschreibung dreier neuer Arten (Hymenoptera: Chrysididae). Linzer biologische Beiträge, 36: 741-760.

Arens W., 2010a. Revision der Hedychridium roseumGruppe in Kleinasien (Hymenoptera: Chrysididae), mit Neubewertung zahlreicher europäischer Taxa und Beschreibung zweier neuer Arten. Linzer biologische Beiträge, 42: 401-458.

Arens W., 2010b. Die Taxa der Hedychridium roseumGruppe auf der Peloponnes (Hymenoptera: Chrysididae), mit Beschreibung einer neuen Art. Linzer biologische Beiträge, 42: 459-476.

Arens W., 2011. Weiterer Beitrag zur Taxonomie und No- 
menklatur griechischer Goldwespen (Hymenoptera: Chrysididae). Linzer biologische Beiträge, 43: 311321.

Arens W., 2012. Notizen zu einigen zentralasiatischen Goldwespen aus der Hedychridium roseum-Gruppe (Hymenoptera: Chrysididae). Linzer biologische Beiträge, 44: 927-932.

Arens W., 2014. Die Goldwespen der Peloponnes (Hymenoptera: Chrysididae) 1. Teil: Die Gattungen Cleptes, Omalus, Holopyga, Hedychrum, Hedychridium und Euchroeus; mit Beschreibung einer neuen Cleptes-Art. Linzer biologische Beiträge, 46: $553-621$

Linsenmaier W., 1959a. Revision der Familie Chrysididae (Hymenoptera) mit besonderer Berücksichtigung der europäischen Spezies. Mitteilungen der Schweizerischen Entomologischen Gesellschaft, 32: 1-232.

Linsenmaier W., 1959b. Revision der Familie Chrysididae (Hymenoptera). Nachtrag. Mitteilungen der Schweizerischen Entomologischen Gesellschaft 32: 233-240.

Kroiss J., Strohm E., van Demben C. \& Vigneron J., 2009. An epicuticular multilayer reflector generates the iri- descent coloration in chrysidid wasps (Hymenoptera, Chrysididae). Naturwissenschaften, 96: 983-986.

Martynova K., 2015. Key for the identification of cuckoo wasps (Hymenoptera Chrysididae) of Eastern Ukraine. Ukrainian Entomological Journal, 5: 33-75. Mohamed Ragaei A.-K., 2015. Role of Color Interference on the Insect's Cuticle Coloration. International Journal of Science and Research, 4: 2306-2314.

Rosa P., Lelej A., Belokobylskij S., Vinokurov N. \& Zaytseva L., 2019. Illustrated and annotated checklist of the Russian cuckoo wasps (Hymenoptera, Chrysididae). Entomofauna, Supplementum, 23: 1-360.

Strumia F. \& Dawah H., 2019. An overview of the Chrysididae (Hymenoptera) of the Red Sea Farasan Archipelago (Saudi Arabia). Journal of Insect Biodiversity, 9: 1-17.

Tyrner P., 1991. Faunistic records from Czechoslovakia. Acta Entomologica Bohemoslovaca, 88: 38-40.

Vinokurov N. 2013. Pecularities in the ecology of cuckoo wasps (Hymenoptera, Chrysididae) in soil biocenoses from the northern macroslope of the Central Caucasus. Proceedings of the Samara Scientific Center of the Russian Academy of Sciences, 15: 1105-1109. 
